\title{
Der mediale Habitus in der frühen Kindheit
}

\author{
Christian Swertz, Gudrun Kern und Erika Kovacova
}

\begin{abstract}
Die empirische Untersuchung der Medienkultur 3- bis 6-jähriger Kindergartenkinder steht im Mittelpunkt des Beitrags. Zum Verständnis der Medienkultur wird der Begriff des medialen Habitus verwendet. Die Ergebnisse zeigen, dass der Begriff des medialen Habitus geeignet ist, um die Medienkultur 3- bis 6-Jähriger zu verstehen, und dass der Ausdruck des medialen Habitus mit einem triangulativen qualitativen Design erfasst werden kann.
\end{abstract}

\section{Einleitung}

Die Nutzung von Medien in der frühen - trotz ihres schon einmal diagnostizierten Verschwindens existierenden - Kindheit, wird in letzter Zeit wieder vermehrt diskutiert. Für die Praxis erfreulich ist, dass die Diskussion sich inzwischen in politischen Vorgaben niedergeschlagen hat (z. B. für Österreich: Ämter der Landesregierungen der österreichischen Bundesländer u. a. 2009). Im wissenschaftlichen Diskurs erscheint dabei unstrittig, dass die mit dem Medienkompetenzbegriff formulierten Ziele nicht für die frühe Kindheit adaptiert werden müssen. Es ist aber erforderlich, die Inhalte anzupassen und entsprechende unterrichtsmethodische Konzepte für den Kindergarten zu entwickeln. Solche Konzepte wurden im Projekt Mediengarten, das im Sparkling Science-Programm des österreichischen Bundesministeriums für Wissenschaft und Forschung gefördert wurde, entwickelt. I Im Mittelpunkt des vorliegenden Beitrags stehen aber nicht die praktischen, sondern die wissenschaftlichen Ergebnisse des Projekts Mediengarten.

In der medienpädagogischen Forschung wird vor allem die Beschäftigung von Kindern und Jugendlichen mit Medien untersucht. Im Mittelpunkt stehen dabei empirisch fundierte Analysen. Dabei liegen derzeit drei Herangehensweisen vor: Aus Sicht der Medienaneignungsforschung wird das Individuum in den Mittelpunkt gerückt (vgl. Wagner u. Theunert 2007). In einer zweiten, häufigeren Sicht wird das Individuum im gesellschaftlichen Kontext gesehen. Dabei wird meist auf den bourdieuschen Habitusbegriff zurückgegriffen. Mit dem Habitusbegriff wird etwa von Iske aus Sicht der virtuellen Ungleichheit die Relation von Feld und Habitus untersucht (vgl. Iske et al. 2007). Paus-Hasebrink rückt in beschreibender Absicht «sowohl die Umweltbedingungen als auch die damit in Interaktion stehenden individuell-subjektiven Bedingungen» (Paus-Hasebrink 2009) in den Mittelpunkt. Niesyto betont in stellungnehmender Absicht die Relevanz der «Förderung von

1 http://www.sparklingscience.at/de/projekte/296-/ 
Kindern und Jugendlichen aus benachteiligten Sozialmilieus» (Niesyto 2009). Und in Beziehung zum Medienkompetenzbegriff gesetzt wird der Ansatz von Bourdieu mit dem Begriff des medialen Habitus bei Biermann (2009) und Kommer (2010), wobei letzterer die bisher umfangreichste und fundierteste Studie zu dieser Perspektive vorgelegt hat.

Im Anschluss an die zuletzt genannten Arbeiten fokussieren wir in einer pädagogisch motivierten Perspektive zunächst das Individuum und verstehen es zugleich im kulturellen Kontext. Dabei sehen wir die Verbindung von empirischen Beschreibungen und theoretischen Überlegungen als konstitutiv für das wissenschaftliche Verstehen an. Dieses kann allerdings nicht neutral bleiben, sondern beinhaltet immer schon normative Momente. Das gilt erst recht, wenn Ergebnisse der pädagogischen Medienforschung in bildender Absicht im Blick auf Medienkompetenz und Mediendidaktik interpretiert werden sollen.

Vor diesem Hintergrund ist die These unserer Studie, dass sich schon in der frühen Kindheit ein medialer Habitus empirisch aufweisen lässt. Um diese These zu begründen, ist zunächst der Begriff des medialen Habitus zu explizieren. Daran anschliessend werden das Untersuchungsdesign und die Ergebnisse vorgestellt. In der Diskussion wird zudem untersucht, ob es erforderlich ist, auch die Mediengenerationentheorie heranzuziehen, um das Medienhandeln von 3- bis 6-Jährigen zu verstehen.

\section{Medialer Habitus}

Für eine Transformation des bourdieuschen Habitusbegriffs in den medienpädagogischen Kontext ist der Blick auf drei Momente erforderlich: Bourdieu schreibt in den feinen Unterschieden: «Aufgabe der Wissenschaft ist die Ermittlung jener Objektivität des Objekts, die sich in der Beziehung zwischen einem Objekt - das bestimmte Anwendungen ermöglicht oder nicht, wie sich freilich erst in seinem sozialen Gebrauch herausstellt (darunter - bei einem technischen Gegenstand der Gebrauch, auf den hin er konzipiert wurde) - und den Einstellungen eines Akteurs oder einer Klasse von Akteuren ergeben; d.h. den Wahrnehmungs-, Bewertungs- und Handlungsschemata, die deren objektive Nützlichkeit im praktischen Gebrauch überhaupt erst konstituieren» (Bourdieu 1982, 171). Bourdieu nimmt damit das System der den Habitus konstituierenden Dispositionen in den Blick. Mit diesem Blick diskutiert er für seine Untersuchung des Geschmacks auch Gegenstände wie Küchen, Inneneinrichtungen und Filme. Dabei rückt Bourdieu in den Blick, inwiefern der Konsument selbst beiträgt zur «Hervorbringung des von ihm konsumierten Produkts» (ebd., 172). Er grenzt sich dabei von den Wirtschaftswissenschaften mit dem Argument ab, dass diese nur die objektiven Eigenschaften betrachten und unterstellen, "dass alle Konsumenten dieselben entscheidenden Attribute wahrnehmen» (ebd., 172). Eben dies sei aber nicht zutreffend, denn, so 
das vorher zitierte Argument, die Wahrnehmung wird im Gebrauch vor dem Hintergrund des Habitus und der ihn konstituierenden Dispositionen erzeugt, und diese Dispositionen sind nicht für alle Menschen gleich, sondern abhängig etwa vom legitimen, bürgerlichen oder populären Geschmack, in denen sich der Habitus ausdrückt.

Dabei unterstellt Bourdieu eine ähnliche Wahrnehmung von vielen Menschen, etwa von all denen, die über einen legitimen Geschmack verfügen. Aus einer medienpädagogischen Sicht, die im Unterschied zur soziologischen Perspektive das Individuum stärker betont, ist es jedoch erforderlich, eher den einzelnen Akteur in den Mittelpunkt zu rücken. Diese Betonung des Individuums ist im Rahmen des Habitusbegriffs möglich und wird von allen eingangs zitierten Arbeiten vorgenommen.

Ein zweites Moment ergibt sich aus der Fussnote, die Bourdieu dem obigen Zitat hinzufügt: «Man könnte sicherlich zeigen, anhand zahlreicher technischer Objekte - wie der von uns untersuchten Photografie - dass mit Ausnahme des in den negativen Bestimmungen, den Grenzen, Implizierten nichts von den technischen Eigenschaften eines Objekts auf dessen sozialen Gebrauch schliessen lässt.» (ebd., 173). Damit ist klar, dass Bourdieu mit der oben zitierten Bemerkung, dass der soziale Gebrauch eines technischen Gegenstandes auch durch den Gebrauch, auf den hin er konzipiert wurde, bestimmt wird, nicht meint, dass das Konzept im Gegenstand zum Ausdruck kommt und dann auch unabhängig vom sozialen Gebrauch oder den Einstellungen von Akteuren besteht.

Das wäre richtig, wenn Medien einen Gebrauch nur bestimmen würden und nicht auch eine bestimmte Verwendung nahelegen oder diese Verwendung immer auch in dem aufgeht, was die Entwickler in dem Gebrauch, auf den der Gegenstand hin konzipiert wurde, ausgedrückt haben - was aber nicht der Fall ist. So haben z. B. die Entwickler von Aufnahme- und Projektionsobjektiven in ihrem Feld die Absicht gehabt, Probleme wie die chromatische und sphärische Abberation zu lösen. Sie haben nicht die Absicht gehabt, die Zuschauer in einen träumerischen Zustand zu versetzen, der, wie Baudry mit Hilfe der lacanschen Psychoanalyse argumentiert hat, durch die primäre Identifikation des Zuschauers mit dem Apparat ausgelöst wird (Baudry 1994, 1068). Die These, dass der träumerische Zustand mit Eigenschaften des Objektivs zusammenhängt, die nicht von konzipierenden Akteuren in ihrem Feld intendiert wurden, sondern mit dem von anderen Akteuren vorgenommenen Transfer der Objektive in andere Felder in Verbindung steht, in denen dann weitere Akteure dem Objektiv eine Bedeutung geben, die schon wegen der fehlenden Kenntnis der konzipierenden Akteure und ihrer Felder vor allem auf die physikalischen Eigenschaften reagiert, ist dabei naheliegend. Bourdieu unterschätzt aus dieser Sicht den physikalischen Gegenstand. Durch diese Lücke in der 
bourdieuschen Theorie droht die Technik zur Ideologie zu werden, weil das medientechnische Apriori (Hartmann 2003, 65) nicht reflektiert werden kann.

Aus einer wirtschaftshistorischen Perspektive liefert Innis einen ähnlichen Einwand, indem er die Effekte technischer Zeichenträger auf die Entstehung von dem, was bei Bourdieu als Dispositiv bezeichnet wird, untersucht hat. Innis zeigt, dass die jeweiligen Kommunikationsmittel Konsequenzen für den Wissenserwerb in den von ihm beschriebenen Epochen hatten (Innis 1997, 69). So argumentiert er z. B., dass der Kodex aus Pergament nicht nur die Verbreitung des Christentums förderte, sondern auch den Geschmack und Stilrichtungen prägte (ebd., 79). Diese Prägung kommt etwa im Übergang vom lauten Lesen zum Lesen mit geschlossenen Lippen zum Ausdruck. Dadurch werden nach Innis Klänge unwichtiger und das Denken wichtiger. Diese Perspektive kann mit Bourdieu nicht eingenommen werden.

Die formulierten Einwände machen auf etwas Offensichtliches aufmerksam: Bourdieu entwickelt keinen Medienbegriff. Die bourdieuschen Begriffe sind allerdings ohnehin ein Problem: In der Bourdieu-Rezeption wird immer wieder betont, dass Bourdieu keine stringenten Definitionen seiner Begriffe liefert. Das ist wenig überraschend, denn Bourdieus Theorie ist eine aus empirischen Beobachtungen emergierende Theorie. Eine solche Theorie ist zweifelsohne wertvoll, wirft aber im Blick auf medienpädagogische Fragestellungen wie z. B. der nach der Begründung der Medienkompetenz das Problem auf, dass der Schluss von einer empirischen Beschreibung auf ein Sollen problematisch ist. Ist etwa die Erziehung zum legitimen Geschmack gut, weil Sie allen Menschen Distinktionsgewinne ermöglicht und damit einen Beitrag zur Verbesserung der Emanzipations- oder Partizipationsmöglichkeiten leistet? Oder ist vielmehr der populäre Geschmack der richtige, weil er ein, wenn auch aus Sicht der herrschenden Elite einfaches, aber doch glückliches und zufriedenes Leben ermöglicht?

Das Übergehen normativer Fragen zeigt sich in einem dritten Moment, an dem deutlich wird, dass Bourdieu einen legitimen Geschmack hat, sich davon aber kaum distanziert. Dass das wohltemperierte Klavier als exemplarisch für den legitimen Geschmack operationalisiert wird, mag sinnvoll sein. Strauss Walzer als charakteristisch für den populären Geschmack zu sehen, ist aber etwas überraschend. Ein Blick auf die französischen Hitlisten der 1950er und 1960er Jahre, in denen Bourdieu seine Studien durchgeführt hat, zeigt jedenfalls, dass er die wesentlichen Objekte populären Geschmacks nicht erfasst hat. Weitere Einwände bezweifeln die Sinnhaftigkeit der Konzepte für aus kulturellen Allesfressern bestehende individualisierte Erlebnisgesellschaften - um einige der zentralen Kritiken zumindest mit Stichwörtern anzudeuten (Übersicht bei Parzer 2008, 40 ff.).

Wegen der genannten Probleme erscheint uns Bourdieus Habitusbegriff nicht unmittelbar als Grundlage für die medienpädagogische Theoriebildung geeignet. Kommer hat diese Probleme gesehen und vorgeschlagen, die bourdieusche The- 
orie weiter zu entwickeln, um sie gegen die genannten Einwände stark zu machen. Er nimmt das Problem der nicht systematisch entwickelten bourdieuschen Begrif$\mathrm{fe}$ in seiner Untersuchung des medialen Habitus zum Anlass, das Habituskonzept in ein fundierteres medienpädagogisches Konzept zu transformieren, indem der Habitusbegriff mit systemtheoretisch-konstruktivistischen Überlegungen in Beziehung gesetzt wird. Dabei wird der Umgang mit Medien als etwas, in dem ein Habitus zum Ausdruck kommt, untersucht: «In den auf die Medien gerichteten (und im Umgang mit diesen sichtbar werdenden) Dispositionen, (Wert-) Zuschreibungen, Klassifikationsschemata und Abgrenzungen - aber auch Kompetenzen und Erfahrungen - spiegelt sich natürlich letztendlich (wie Bourdieu ja eindrücklich in den «Feinen Unterschieden» zeigt) der Habitus (bzw. sind sie ein Ausdruck von diesem)» (Kommer 2010, 92). Diese Sichtweise erscheint uns als fruchtbar, denn zum einen bleiben mit den auf Medien gerichteten Dispositionen der Akteure auch soziale Strukturen im Blick, zum anderen wird aus unserer Sicht mit der Differenz von Dispositionen und Ausdruck zugleich die Möglichkeit und Grenze der empirischen Untersuchung des Habitus markiert (vgl. Iske u. Swertz 2005).

Allerdings bleibt die Fundierung des Habitusbegriffs in der systemisch-konstruktivistischen Theorie bei Kommer etwas unscharf, weil auf der einen Seite Ähnlichkeiten gezeigt werden, diese aber nicht in Passungen überführt werden, und zugleich immer wieder auf nicht näher spezifizierte fundamentale Unterschiede zwischen Habituskonzept und struktureller Koppelung aufmerksam gemacht wird. Eine theoretische Fundierung des Habituskonzepts wird damit nicht erreicht und ist von Kommer auch nicht beabsichtigt. Vielmehr wird der Habitus als «theoriegeleitetes Analysekonzept» (Kommer 2010, 92) verstanden. Dabei ist der Ausdruck Analysekonzept nicht im methodologischen Sinne gemeint, sondern deskriptiv. Es wird also eine Beschreibung medialen Handelns erreicht, die deutlich macht, dass Menschen im Blick auf den Umgang mit Medien in Kindheit und Jugend Gewohnheiten entwickeln, die einen erheblichen Einfluss auf die Mediennutzung in ihrem weiteren Leben haben. Diese Gewohnheiten beeinflussen offenbar auch das Handeln in Bildungsinstitutionen.

Da wir eine theoretische Fundierung des Begriffs des medialen Habitus für relevant halten, ist es damit erforderlich, den Begriff des medialen Habitus weiter zu entwickeln. Das kann hier nicht in der erforderlichen Breite erfolgen. Daher beschränken wir uns darauf, den Begriff des medialen Habitus in Bezug zum Medienbegriff zu setzen.

Medien verstehen wir als die Relationen von materiellen Zeichenträgern, Zeichen und Subjekten, in denen materielle Zeichenträger von Subjekten als Zeichen verwendet werden (vgl. Swertz 2009). Medien bestehen aus dieser Sicht stets aus der materiellen Welt zugehörigem materiellem Substrat und der immateriellen Zeichenwelt zugehörigen Zeichen. Alle Beziehungen dieser Elemente aufeinander im 
Medium werden von Subjekten produziert und rezipiert. Das Subjekt kann dabei seine mediale Freiheit, die es machtförmig, d.h. in einem auch selbstermächtigenden Akt, gewinnen kann, in Auseinandersetzung mit der zumindest aus Sicht eines begrenzt gedachten Menschen unbegrenzten Kombinierbarkeit von Zeichen gewinnen. Insofern es sich nur so zu seinem Habitus in ein Verhältnis setzen kann, ist klar, dass der Habitus selbst wieder als im Raum des Medialen verortet zu denken ist. Um ein Medium handelt es sich beim Habitus nicht, weil der Habitus eine Bedeutung von Zeichen ist. Der Habitus ist in den bewussten oder unbewussten Relationen des Subjekts zu immateriellen Zeichen und materiellen Zeichenträgern zu verorten, die machtförmig angeeignet werden können.

Damit können wir hier eine erste Bestimmung des Begriffs des medialen Habitus vorschlagen: Der mediale Habitus ist der Ausdruck des von Menschen bewusst und unbewusst wahrgenommenen Eindrucks, den Medien im Menschen hinterlassen haben. Eindruck und Ausdruck erfolgen bewusst und unbewusst. Da der mediale Habitus sich nicht unmittelbar, sondern nur mittelbar im Geschmack äussert, kann er nur anhand der Beschreibung des Eindrucks und des Ausdrucks rekonstruiert werden. Wird mit dem medialen Habitus dieses Konstrukt bezeichnet, ist also die empirische Beschreibung des Eindrucks oder des Ausdrucks gemeint. Der Habitus selbst ist nicht dieses Konstrukt, er kann daher nicht empirisch, sondern nur theoretisch erfasst werden. Da nur mittelbare Beziehungen zwischen der Theorie und der Empirie hergestellt werden können - schon weil auch für das Herstellen dieser Beziehung Medien erforderlich sind und das Konstrukt daher ebenfalls dem Ausdruck des Eindrucks von Medien unterliegt - bleibt die empirische Beschreibung notwendig unscharf. Die bewusste Gestaltung des Ergebnisses des Eindrucks (Mediendidaktik) und die dichte Interpretation des Eindrucks (Medienund Rezeptionsforschung) sind allerdings möglich.

Dabei scheint es uns erforderlich, den Begriff des medialen Habitus in medienpädagogischer Absicht nicht nur deskriptiv zu verwenden, sondern durchaus auch normativ durch eine Orientierung am «semantischen Schnitt» (Meder 1998) im Interesse der Möglichkeit der Freiheit (Reichenbach 2001) aufzuladen. Dies kann hier nur angedeutet werden.

Wichtig ist hier, die entscheidende Differenz zur bourdieuschen Konzeption noch einmal deutlich zu machen: Wenn z. B. die Distinktionen und die damit erzeugbaren Distinktionsgewinne auch mit bestimmten Medien zu tun haben, etwa der Gegenüberstellung von Buchdruck und Fernsehen, bei der das Fernsehen als Unterhaltungsmedium den Massstäben herrschender Eliten nicht gerecht wird (Mikos 2007), dann ist es wenig plausibel, dies unabhängig von der medienspezifischen Form der Verständigung über kulturelle Gehalte zu sehen. Daher verstehen wir den medialen Habitus nicht nur als durch Felder strukturierte Struktur, sondern auch als durch materielle Zeichenträger strukturierte Struktur (Swertz 2003a, 2003b, 2004). 
Dies ist nicht nur Ausdruck ökonomischen Kapitals, das sich in Medienbesitz niederschlägt, sondern es meint auch die Strukturierung des Habitus durch die materiellen Zeichenträger, was McLuhan (1994) als heisse und kalte Kultur in Bezug auf kalte und heisse Medien beschrieben hat.

Mit dem Begriff des medialen Habitus wurde im Projekt Mediengarten versucht, die Medienkultur von 3- bis 6-Jährigen zu verstehen. Bisherige Studien der Medienforschung zeigen auf, dass die Alltagswelt von älteren Kindern wesentlich durch die Nutzung von Fernsehen und Musik und zunehmend auch von digitalen Spielen geprägt ist (Spanhel 1990, Leu 1993, Glogauer 1993, Süss et al. 1998, Aufenanger u. Gerlach 2005, Fromme et al. 2000, Fehr u. Fritz 1995). Durch den Wandel der medialen Angebote kommt es dabei zu einer ständigen Veränderung der Alltagswelt. Theorien zu einem diesen Wandel berücksichtigenden Verständnis des Medienalltags von Kindern wurden bisher vor allem im Kontext der Mediensozialisationstheorie entwickelt (Vollbrecht, Ferchhoff u. Baacke 1997, Fritz u. Fehr 1997). Mit dem Begriff der Selbstsozialisation wird dabei in den Mittelpunkt gerückt, dass Kinder dazu in der Lage sind, sich die Medienwelt selbst zu erschliessen und in einer spezifischen Art und Weise anzueignen. Dabei werden jedoch 3- bis 6-Jährige als eine für die Mediensozialisation wichtige Altersgruppe nur selten thematisiert und der mediale Habitus nicht berücksichtigt.

\section{Methode}

Methodisch wurde im Projekt Mediengarten eine qualitative Untersuchung 3- bis 6-Jähriger in einer Kooperation mit 151 Schülerinnen und Schüler durchgeführt, die Bildungsanstalten für Kindergartenpädagogik (Bakip) im letzten oder vorletzten Ausbildungsjahr besuchen. Bakips sind Schulen, in denen in einer vierjährigen zweiten Sekundarstufe (8.-12. Schulstufe) sowohl die Zugangsberechtigung zum tertiären Sektor als auch die Berufsausbildung zur Kindergartenpädagogin bzw. zum Kindergartenpädagogen vermittelt wird. Das Projekt wurde vom Sparkling Sciences- Programm des österreichischen Bundesministeriums für Wissenschaft und Forschung gefördert.

Die Kooperation mit Bakips hatte den Vorteil, dass die Datenerhebung durch Personen durchgeführt werden konnte, die in Kindergärten tätig und mit den befragten Kindern vertraut sind. Zur Vorbereitung der Schülerinnen und Schüler wurden drei ganztägige Workshops durchgeführt, in denen theoretische und methodische Grundlagen vermittelt sowie praktische Übungen zur Datenerhebung und -auswertung durchgeführt wurden. Gegenstand des Projekts war dabei nicht nur die Erhebung und Auswertung der Daten, sondern auch die Erprobung von Verfahren der Datenerhebung.

Das Projekt greift methodologisch auf das von Bohnsack (2003) entwickelte Konzept zurück. Bohnsack bezeichnet den Interpretationsprozess, in dem die Differenz 
zwischen Forschenden und Untersuchten berücksichtigt wird, als Fremdverstehen. Methodologisch kontrolliertes Fremdverstehen ermöglicht eine wissenschaftliche Rekonstruktion der beobachteten Alltagspraxis. Das Ziel ist dabei die Rekonstruktion der Relevanzsysteme der untersuchten Menschen (Bohnsack 2003, 20ff.). Das Projekt Mediengarten zielte auf die Rekonstruktion des medialen Habitus von 3- bis 6-Jährigen in der Absicht ab, die Relevanzsysteme, auf die die Kinder sich beziehen, als Ausdruck ihres medialen Habitus zu explizieren.

Neben der Rekonstruktion von Relevanzsystemen ist nach Bohnsack ein reflexives Verhältnis der Forscherinnen und Forscher zum Forschungsprozess im Sinne der Rekonstrukion der Prozesse des Erkennens ein zentrales Moment qualitativer Sozialforschung. Die Selbstreflexion der Forscherinnen und Forscher wird damit zu einem Moment des Forschungsprozesses. Dieses Moment hatte im Projekt Mediengarten eine doppelte Relevanz, da diese Rekonstruktion der Rekonstruktion nicht nur für den Forschungsprozess bedeutsam war, sondern auch eine Reflexion des eigenen Handelns der Forscherinnen und Forscher implizierte. Damit wurde es für Schülerinnen und Schüler als Forschende wichtig, ihr eigenes Medienhandeln zu reflektieren.

Als erste Datenerhebungsmethode wurden die Schülerinnen und Schüler daher gebeten, ein Forschungstagebuch im Sinne eines Forschungsprotokolls zu führen. Diese Forschungstagebücher wurden analog zu den Vorbereitungs-, Durchführungs- und Reflexionsberichten erstellt, die den Schülerinnen und Schülern als Teil ihrer Ausbildung bekannt waren. Als zweite Datenerhebungsmethode wurden Interviews mit den Kindern durchgeführt. Diese wurden im Anschluss an medienpädagogische Projekte durchgeführt, sodass die Medienthematik den Kindern bereits präsent war. Als dritte Datenerhebungsmethode des verwendeten Mehrmethodendesigns wurden Bilddaten erhoben. In der qualitativen Forschung wird bisher eher selten auf Bilddaten wie Kinderzeichnungen zurückgegriffen (Neuß 2000). Neuß macht darauf aufmerksam, dass es von vorneherein zwei Möglichkeiten gibt, die Kinderzeichnung in den Forschungsprozess einzubinden. Zum ersten geben die Zeichnungen eine Möglichkeit ein Gespräch mit dem Kind zu beginnen, zum anderen können Zeichnungen eine Interpretation der subjektiv wahrgenommenen Medienwirklichkeit der Kinder ermöglichen. Im Projekt Mediengarten wurde auf beide Möglichkeiten zurückgegriffen.

Durch die unterschiedlichen Erhebungsmedien (Textdaten, Bilddaten) wird ein kontrastierendes Element in die Untersuchung eingezogen, das eine triangulierende Validierung im Zuge der Interpretation ermöglicht. Da Datenerhebungsmethoden in der qualitativen Forschung im Sinne der rekonstruktiven Rekonstruktion zugleich Gegenstand der Forschung sind und zudem nur wenige Erfahrungen mit der Datenerhebung bei 3- bis 6-Jährigen vorliegen, wurden die Schülerinnen und Schüler auch darum gebeten, ausgehend von ihren mehrjährigen Erfahrungen in 
der Arbeit mit Kindern Vorschläge für weitere Datenerhebungsmethoden zu erarbeiten, deren Brauchbarkeit anhand der Reflexionsbögen sowie der Interpretierbarkeit der erhobenen Daten beurteilt wurde.

Die Auswertung der Textdaten erfolgte in Anlehnung an Bohnsack in einem sechsstufigen Verfahren, wobei der erste Schritt durch die Schülerinnen und Schüler durchgeführt wurde. Zunächst wurden die Daten von den Schülerinnen und Schülern transkribiert. Im zweiten Schritt erfolgte eine formulierende Interpretation (Bohnsack 2003, 134) im Forschungsteam. Diese Interpretation verblieb im Bereich des immanenten Sinngehalts der Interviews. Im dritten Schritt wurde die formulierende Interpretation der Interviews durch eine ebenfalls formulierende Interpretation der Kinderzeichnungen im Sinne einer qualitativen Bildinterpretation (Bohnsack 2003, 155 ff.) ergänzt. Im vierten Schritt wurden die beiden formulierenden Interpretationen miteinander verglichen. Im fünften Schritt wurde die reflektierende Interpretation der Daten vorgenommen. Dieser Schritt bildete die Grundlage für die Rückbindung der Daten an die theoretische Fragestellung des Projekts. Im Anschluss an die Datenauswertung wurde im sechsten Schritt die Rückbindung der Daten an die Theorien des medialen Habitus und der Mediengeneration vorgenommen.

Als Auswertungsverfahren für die Bilddaten wurden sequentielle Bildanalysen und in toto Bildanalysen verwendet. Die Bilddaten wurden von Interpretationsgruppen zunächst ohne Kenntnis der Textdaten interpretiert. Erst im letzten Schritt wurden die Interpretationen der Bild- und Textdaten zusammengeführt.

Insgesamt waren 151 SchülerInnen an sechs Bakips beteiligt. Das Zusammenführen und Bereitstellen der Datensätze (Transkription, Bildmaterial und Vorbereitungsoder Reflexionsbericht) ist 101 Schülerinnen und Schülern gelungen. Ein wesentliches Hindernis waren technische Probleme, die durch die unerwartet geringe Kompetenz der Schülerinnen und Schüler im Bereich der Medienkunde verursacht wurden. So konnten einige Schülerinnen und Schüler keine Audioaufzeichnungen mit ihren Handys anfertigen, andere konnten diese nicht auslesen oder keine Handyfotos in ausreichender Qualität erstellen. Darüber hinaus war die Handhabung der Dateien für die Schülerinnen und Schüler ein Problem, was in manchen Fällen zu Datenverlust geführt hat.

Insgesamt wurde auswertbares Material in folgenden Fallzahlen eingereicht, die hier zum Zwecke der Darstellung der Stichprobe nach Alter, Geschlecht und Migrationshintergrund der Kinder aufgeschlüsselt werden:

1. Jungen (3-4 J): 7 Fälle

2. Jungen (5-6 J): 14 Fälle

3. Jungen mit Migrationshintergrund (5-6 J): 3 Fälle

4. Mädchen (3-4 J): 8 Fälle 
5. Mädchen (5-6 J): 13 Fälle

6. Mädchen mit Migrationshintergrund (5-6 J): 5 Fälle

Damit konnten 50 Fälle interpretiert werden. Für die Analyse jeden Falls wurden die Vorbereitungsbögen, das Bildmaterial, die Interviews sowie die Reflexionen der Schülerlnnen ausgewertet. Wegen des hohen Aufwands insbesondere der Auswertung des Bildmaterials und der Relationierung der Daten war es erforderlich, aus den verfügbaren Fällen eine Zufallsstichprobe zu ziehen, um die zu analysierende Fallanzahl auf eine mit den forschungspraktisch verfügbaren Ressourcen auswertbare Fallanzahl zu bringen. Insgesamt wurden für die folgenden Ergebnisse 13 Fälle ausgewertet.

\section{Ergebnisse}

\subsection{Methodologie}

Im Blick auf die erprobten Datenerhebungsverfahren ist zunächst festzuhalten, dass Handpuppeninterviews sich nicht bewährt haben. Dieses Verfahren konnte in unserer Studie kaum dazu beitragen, relevante Aussagen von den Kindern über ihre Mediennutzung zu erhalten. Unklar bleibt die Verwendung von Plastilin oder von Puppenhäusern, da die in diesem Projekt erhobenen Daten über diese beiden Erhebungsverfahren keine hinreichend genauen Aussagen ermöglicht haben.

Als relevant hat sich die Verwendung von Interviews in Verbindung mit Collagen oder mit Kinderzeichnungen erwiesen. Für die Kinderzeichnungen ist festzuhalten, dass die Interviews nicht während der Entstehung der Kinderzeichnung, sondern erst danach geführt werden sollten. Gleichwohl ist es sinnvoll, auch schon den Entstehungsprozess der Zeichnung zu dokumentieren, weil damit die Interpretation orientiert werden kann. Mit Kinderzeichnungen können detailliertere Aussagen aber nur über einige wenige Medien erhoben werden. Die ganze Breite der von Kindern genutzten Medien kann nicht erhoben werden, da die Anfertigung der Zeichnung dann schnell komplex wird und zu lange dauert. Damit sind Kinderzeichnungen vor allem dazu geeignet, die von Kindern als dominant wahrgenommenen Medien zu erfassen.

Collagen haben sich als Datenerhebungsverfahren gut bewährt. Dabei haben sich Collagen vor allem als gut dazu geeignet erwiesen, die Breite des medialen Raums, in dem Kinder sich bewegen, zu erfassen. Allerdings ist die Vorbereitung von Collagen als Datenerhebungsverfahren anspruchsvoller als die Arbeit mit Kinderzeichnungen, da durch die Auswahl des Materials, aus dem die Collage erstellt wird, eine Vorentscheidung getroffen wird, deren suggestive Effekte im Erhebungsdesign berücksichtigt werden müssen. Wenn aber eine genügend grosse Auswahl an Bildern von Medien bereitgestellt wird, kann insbesondere in Verbindung mit Hausskizzen oder -modellen die Wahrnehmung der räumlichen 
Lokalisierung von Medien durch die Kinder erfasst werden. Im Falle von Collagen ist es auch möglich, während der Erstellung der Collage vertiefende verbale Daten zu erheben. Mit Blick auf die Auswertung ist eine genaue und umfassende Dokumentation der zur Verfügung gestellten Materialien erforderlich.

\subsection{Medialer Habitus}

Der mediale Habitus wird von Kommer in enger Anlehnung an Bourdieu als strukturierende und strukturierte Struktur beschrieben, die als inkorporierte Disposition zu verstehen ist. Dabei wird insbesondere die Familie als ein durch verfügbares Kapital bestimmter sozialer Ort gesehen, an dem der Habitus erworben wird (Kommer 2009: 60ff.).

Durch die hier untersuchte Stichprobe ist zunächst zu berücksichtigen, dass in unserer Untersuchung die Phase der familiären Primärerziehung dargestellt wird. Es wäre anzunehmen, dass die Inkorporation kulturellen Kapitals bei 3- bis 6-Jährigen noch nicht zu stabilen Dispositionen geführt hat.

Unsere Untersuchung hat zunächst gezeigt, dass es bei 3- bis 6-Jährigen in Österreich weniger das ökonomische Kapital ist, dass für die Entstehung des medialen Habitus relevant ist. Den Kindern stehen durchwegs alle Medien zur Verfügung. Auch die Länge von finanzierbaren Ausbildungsgängen spielt noch keine Rolle. Zudem bestimmt, wie der Ausdruck des medialen Habitus der Kinder zeigt, die Medienausstattung nicht die Nutzung. Es gibt z. B. Kinder, die viele Bücher im Kinderzimmer haben, bei denen aber die Bücher kaum mehr als die Funktion haben, den Fernseher einzurahmen. Die beobachteten Unterschiede, etwa mit Blick auf den Besitz eines eigenen Fernsehers, sind in diesem Sinne weniger auf Restriktionen, die durch das verfügbare ökonomischen Kapital erklärt werden können, und mehr auf Restriktionen, die in Zusammenhang mit dem kulturellen Kapital der Eltern stehen, zurückzuführen.

Im Ausdruck des medialen Habitus der Kinder kam entgegen unserer Erwartung deutlich die Inkorporation kulturellen Kapitals zum Ausdruck. Als wichtiges Moment hat sich dabei der Geschmack erwiesen, der in der Gerätewahl zum Ausdruck kommt. Die Inkorporation stellt sich dabei allerdings noch widersprüchlich dar. Das wird besonders bei den buch- und audioorientierten Familien deutlich: Hier werden zwar die skeptischen Urteile der Eltern über audiovisuelle Medien von den Kindern reproduziert; die geäusserten eigenen Präferenzen der Kinder rücken aber genau dieses Segment in den Mittelpunkt. Das ist nicht zuletzt im Zusammenhang mit der Einschränkung von Kindern zu sehen, die noch nicht lesen können: Während auf der einen Seite das Fernsehen die Möglichkeit einer eigenständigen Nutzung eröffnet, wird es auf der anderen Seite von den Kindern aus buch- und audioorientierten Familien als zumindest potentiell defizitäres Medium beschrieben. Hier drängt sich die These auf, dass es genau diese Spannung ist, die als 
von den Kindern wahrgenommene Differenz zum einen zu einem Bildungsanlass werden kann und die zum anderen als Index des Inkorporationsprozesses verstanden werden kann. Diese Vermutung kann auch damit gestützt werden, dass es bei manchen Kindern ein Bestandteil des medialen Habitus ist, dass die Nutzung von Medien oder zumindest von bestimmten Medien begründungsbedürftig ist. Ein weiterer Beleg kann in dem Umstand gesehen werden, dass manche Kinder, die in buchorientierten Familien aufwachsen, einen eher statisch-visuellen Geschmack zum Ausdruck gebracht haben und Fotos bevorzugen, während einige der untersuchten Kinder aus buchfernen Familien einen dynamisch-visuellen Geschmack aufweisen und Videos bevorzugen.

Unterschiede wurden auch im verfügbaren objektivierten Kulturkapital sichtbar. Dieses wird zunächst kaum aktiv von den Kindern ausgewählt. Nach ihren Wünschen mit Blick auf Medien gefragt, haben die Kinder fast ausschliesslich Wünsche bezüglich des Besitzes von Geräten, aber kaum Wünsche bezüglich bestimmter Inhalte geäussert. Es wurde aber deutlich, dass die Inhalte von den Kindern durchaus wahrgenommen werden und diese Wahrnehmung auch ausgedrückt wird. Dieser Ausdruck zeigt, dass die Auswahl der Inhalte im Wesentlichen durch die Familie (Eltern und ältere Geschwister) und nur ansatzweise durch Freundinnen und Freunde bestimmt wird. Allerdings wird das verfügbare objektivierte Kulturkapital von den Kindern selbst offenbar kaum für das Erzeugen von Distinktionsgewinnen verwendet. Ein höherer Status wird vielmehr über Geräteästhetik und Quantitäten erzeugt: In ästhetischer Hinsicht scheint das Äussere der Geräte etc. einen höheren Stellenwert einzunehmen als der Inhalt: Cooles Design ist wichtig, ein grosser Fernseher liefert einen höheren Status als ein kleiner, viele CDs zu haben ist besser als wenig CDs zu haben, viele Bücher sind besser als wenig. In einer ganzen Reihe von Fällen wurde dabei von den Kindern eine Sättigung zum Ausdruck gebracht: Längst nicht alle Kinder haben das Verlangen nach einer höheren Quantität an Datenträgern. Vielmehr sind es oft nur einige wenige Inhalte, die von den Kindern dominant wahrgenommen, berichtet und ausgewählt werden. Alle darüber hinausgehenden Inhalte, die von den Kindern durchaus rezipiert werden, werden von den Eltern oder älteren Geschwistern bestimmt. Insofern erfolgt die Inkorporation objektivierten Kulturkapitals in diesem Alter eher fremd- als selbstgesteuert.

In der Wahrnehmung der regelförmigen Fremdsteuerung durch die Kinder kommt dabei eine eigene Ambivalenz zum Ausdruck: Über die Verwendung von Büchern dürfen alle Kinder selbst bestimmen, können es aber mangels Lesekompetenz meist nicht. Über Fernseher und Computerspiele könnten die Kinder selbst bestimmen, dürfen es aber meist nicht. Während der erste Fall klar ist, sind am zweiten Differenzen des Geschmacks festzumachen, die durch die Eltern bestimmt werden. Interessant ist in diesem Zusammenhang auch der in Einzelfällen geäusserte Wunsch nach der eigenen Inszenierung auf Fotos (z. B. geäussert in der Relevanz 
von Kleidung). Daran wird deutlich, dass bereits ein objektiviertes kulturelles Kapital inkorporiert worden ist, und zudem ein erstes Bewusstsein für die Differenz von Person und medialer Darstellung besteht.

Eine weitere Tendenz, die zum Ausdruck kommt, ist die Individualisierungstendenz. Es geht vielen Kindern nicht darum, überhaupt fernsehen zu dürfen, sondern darum, ein eigenes Gerät für den persönlichen Gebrauch zur Verfügung zu haben. Das kann ein Handheld, eine Spielkonsole oder ein Mobiltelefon sein; entscheidend ist in jedem Fall die individuelle Verfügbarkeit des Geräts.

Die Analyse der Kinderzeichnungen hat gezeigt, dass die Kinder mitunter Medien als dominant wahrnehmen, die sie selbst überhaupt nicht verwenden. Das gilt z.B. für Mobiltelefone, die als wichtig wahrgenommen werden, den Kindern in der Regel aber nicht zur Verfügung stehen. Im Blick auf den medialen Habitus ist festzuhalten, dass die Kinder sich damit als eingeschränkt wahrnehmen und ihnen das auch bewusst ist. Zugleich wird deutlich, dass zumindest für Vorschulkinder sich über die Begrenzung des Medienzugangs eine Form der Konstruktion von Kindheit andeutet.

\section{Diskussion}

Eine Ausgangsfrage des Projekts Mediengarten war die Frage, ob der Begriff der Mediengeneration oder der Begriff des medialen Habitus besser geeignet ist, um die Medienkultur 3- bis 6-Jähriger zu verstehen. Da im Zuge der Datenauswertung deutlich wurde, dass der Begriff des medialen Habitus sinnvoller ist, wurde dieser in den Mittelpunkt des Beitrags gerückt. Im Folgenden wird nun ergänzend der Begriff der Mediengeneration diskutiert.

Im Blick auf die Beschreibung generationsspezifischer konjunktiver Erfahrungsräume hebt Schäffer (2003) in seiner Generationentheorie die Differenz zwischen generationsspezifischen Medienpraxiskulturen und intergenerationellen Bildungsprozessen hervor. Dabei wird vor allem der Funktion der Kommunikationskultur eine besondere Bedeutung beigemessen. Schäffer arbeitet aus seinen Daten heraus, dass in der Kommunikation über intergenerationelle Differenzerfahrungen Bildungsprozesse angestossen werden, «die sich im Modus der Begegnung unterschiedlicher konjunktiver Erfahrungsräume entfalten» (Schäffer 2003, 326).

Die vorliegende Untersuchung legt nahe, dass die Beobachtungen zwar ebenfalls als das Anstossen von Bildungsprozessen durch intergenerationelle Differenzerfahrungen verstanden werden können, dass dabei aber keine Begegnung unterschiedlicher konjunktiver Erfahrungsräume zu beobachten ist. Vielmehr können die beobachteten Erfahrungen treffender als Erziehungsverhältnis zwischen Eltern und Kindern verstanden werden.

Dabei ist zunächst daran zu erinnern, dass Schäffer seine Studie 2003 veröffentlicht hat. Seine Daten wurden in Deutschland im Jahr 2000 erhoben. Zu diesem 
Zweitpunkt waren erstens die Auflösung des eisernen Vorhangs und zweitens die massenhafte Verbreitung des Internets noch relativ neue Entwicklungen, die, insbesondere was die Verbreitung des Internets angeht, die Medienkultur noch nicht in dem Masse durchdrungen hatten wie das heute der Fall ist. Diese von Schäffer ausführlich reflektierte Situation drückt sich in seinen Ergebnissen deutlich aus.

Die von Schäffer beschriebenen konjunktiven Erfahrungsräume und die damit verbundenen Generationenlagerungen sind für unsere Studie anders darzustellen. Zunächst war Österreich 1990 von der Aufhebung des eisernen Vorhangs in anderer Weise betroffen als Deutschland. Ganz unterschiedliche medienkulturelle Erfahrungsräume, wie sie in der BRD und der DDR bestanden, spielen daher nicht in die Ergebnisse hinein. Die untersuchten Generationenlagerungen sind in dieser Hinsicht vielmehr als wesentlich homogener anzusehen. Darüber hinaus kommt bei Schäffer noch eine deutliche Distanz der Elterngeneration zur Computertechnologie zum Ausdruck. Davon kann heute kaum noch die Rede sein: Die Elterngeneration, die untersuchten Schülerlnnen und deren Lehrerlnnen nutzen Computertechnologie gleichermassen und ganz selbstverständlich im Alltag, wenn auch nicht in der gleichen Art und Weise.

Zuletzt ist zu berücksichtigen, dass die jüngsten Befragten bei Schäffer Jugendliche waren. Jugendlich befinden sich aber schon in der Phase der Ablösung vom Elternhaus und verfügen über die Fähigkeit zum vollgültigen Rollenhandeln. Beides ist bei 3- bis 6-Jährigen nicht der Fall. Daher erscheint es als sinnvoller, die vorliegenden Daten mit dem Begriff des medialen Habitus zu interpretieren.

Zunächst machen unsere Analysen deutlich, dass der mediale Habitus der Kinder nicht durch eine abwertende Distanz zu den Eltern bestimmt ist. Es kann aber auch keine Rede davon sein, dass keine Distanz vorhanden ist. Diese kann durch folgende Momente beschrieben werden:

1. Der mediale Raum wird von den Kindern in ihrer medienkulturellen Selbstwahrnehmung als durch instrumentell-qualifikatorische Grenzen beschränkt wahrgenommen. Sie können nicht lesen und daher keine Textbücher verwenden, sie können keine Computersoftware installieren etc.

2. Die Kinder beschreiben im Ausdruck ihres medialen Habitus ihren Medienraum als durch die Eltern beschränkt. Das drückt sich in den von den Kindern berichteten Regeln zur Mediennutzung aus. Dominant ist für die Kinder nicht die eigene Bestimmung, sondern das Bestimmt werden durch die Eltern.

3. Aus Sicht der von Schäffer beschriebenen Nähe-Distanz- Dimension wird deutlich, dass Medien von den Eltern auch dazu verwendet werden, Distanz zu den Kindern aufzubauen. Im Ausdruck des medialen Habitus der Kinder äussert sich das darin, dass Medien gelegentlich als Störgrössen in der Beziehung zu den Eltern wahrgenommen werden. Wenn die Eltern lesen, fernsehen oder am Computer tätig sind, sind sie für die Kinder nicht ansprechbar. 
Insbesondere die letzten beiden von den Kindern wahrgenommenen Distanzierungen können als Ausdruck der Konstruktion eines medialen Raumes verstanden werden. So gesehen kann die Medienkultur der Kinder weniger als eine Generationendifferenz denn als ein medialer Kindheitsraum verstanden werden, der von den Eltern konstruiert wird. Diese Konstruktion wird von den Kindern wahrgenommen und findet sich im Ausdruck ihres medialen Habitus wieder. Im Mittelpunkt steht für die Kinder dabei das Ziel, die Mediennutzung so wie die Eltern selbst bestimmen zu können. Erwachsen sein sein heisst für 3- bis 6-Jährige klar: Die Medien benutzen dürfen, die die Grossen benutzen - seien es ältere Geschwister, seien es Eltern. Dieses "Gross sein» wird von den Kindern an verschiedenen Momenten festgemacht. Am wichtigsten scheint den Kindern zu sein, dass sie bestimmte Medien bedienen können. Wenn Sie Medien bedienen können, ist es für sie zum Teil nicht nachvollziehbar, warum sie diese dann nicht benutzen dürfen. Sie nehmen sich selbst als Menschen war, die mittels des Verfügens über Medientechnik ihren Platz in der sozialen Gemeinschaft einnehmen. Dieser Platz wird in der Wahrnehmung der Kinder durch die Regeln der Mediennutzung von den Eltern bestimmt. Möglicherweise ist es sinnvoller, dieses Verhältnis weniger als Generationendifferenz im soziologischen Sinne und eher als Generationenverhältnis im pädagogischen Sinne zu verstehen. Hier könnte - aber das kann hier nur als These angedeutet werden - das pädagogische Verständnis des Generationenverhältnisses, das über Dilthey und Mannheim in einer soziologisch reformulierten Form zur Grundlage von Schäffers Arbeit geworden ist, als Verstehenshorizont herangezogen werden.

Während die Generationendifferenz in einer anderen Form als bei Schäffer zum Ausdruck kommt, stellt sich der Generationenzusammenhang ähnlich dar. Es wird deutlich, dass allen Kindern ein breites Ensemble an Medien zur Verfügung steht und von diesen im Ausdruck ihrer medienkulturellen Selbstwahrnehmung auch entsprechend beschrieben wird. Allerdings sind dabei deutliche individuelle Differenzen festzustellen, die mit dem Begriff des medialen Habitus verstanden werden können.

Mit Blick auf den Geschmack ist klar, dass die auf quantitativen empirischen Daten aus den 1960er Jahren basierende Differenzierung von Bourdieu so nicht mehr sinnvoll verwendet werden kann. Zudem ist anzunehmen, dass Kinder, die aus Familien mit legitimem Geschmack stammen, nicht in der von uns untersuchten Stichprobe vertreten waren. In einer groben Annäherung könnte der in unserer Stichprobe erhobene mittlere Geschmack mit einer Bevorzugung auditiver Medien und Printmedien verbunden werden; der populäre Geschmack mit einer Bevorzugung audiovisueller Medien. Damit käme der Geschmack allerdings nicht wie bei Bourdieu in inhaltlichen Präferenzen, sondern in Präferenzen für bestimmte 
Medientechniken zum Ausdruck. Das mag mit der untersuchten Altersgruppe in Zusammenhang gebracht werden und erfordert eine hier nicht zu leistende entwicklungspädagogische Reflexion. Zudem ist die Heterogenität der von den Kindern berichteten Ausdrücke recht gross, die Stichprobe dagegen eher klein. Wegen dieses Umstandes verbietet sich eine Typenbildung aus forschungsmethodischen Gründen. Allerdings wäre zu vermuten, dass die Anzahl an möglichen Kombinationen, die sich aus den vorgenommen Klassifizierungen ergibt, nicht so hoch ist, dass die volle Komplexität in einer quantitativen Studie nicht abbildbar wäre. Zugleich wird deutlich, dass zumindest für Vorschulkinder sich über die Begrenzung des Medienzugangs eine Form der Konstruktion von Kindheit andeutet. Das kann allerdings kaum mit dem Habitusbegriff erfasst werden, sondern verweist auf einen pädagogischen Generationenbegriff.

Damit kann festgehalten werden, dass der Begriff des medialen Habitus und das verwendete Untersuchungsdesign sich für die Entwicklung eines Verständnisses der Medienkultur von 3- bis 6-Jährigen bewährt haben. Darüber hinaus hat sich im Rahmen des Gesamtprojekts gezeigt, dass die Theorie des medialen Habitus auch dazu geeignet ist, die medienbezogene Selbstreflexion von Schülerinnen, Schülern, Lehrerinnen und Lehrern anzuregen und zum Ausgangspunkt für die Medienkompetenzvermittlung im Kindergarten zu machen. Über die dazu im Projekt entwickelten Module wird an anderer Stelle zu berichten sein.

\section{Literatur}

Ämter der Landesregierungen der österreichischen Bundesländer, Magistrat der Stadt Wien, Bundesministerium für Unterricht, Kunst und Kultur. 2009. «Bundesländerübergreifender BildungsRahmenPlan.» http://www.bmukk.gv.at/medienpool/18698/bildungs rahmenplan.pdf.

Aufenanger, Stefan und Franz Gerlach. 2005. «Vorschulkinder und Computer: Sozialisationseffekte und pädagogische Handlungsmöglichkeiten in Tageseinrichtungen für Kinder.» http://www.lpr-hessen.de/files/130105.pdf, 29.11.2007.

Baacke, Dieter, Wilfried Ferchhoff, Ralf Vollbrecht. 1997. "Kinder und Jugendliche in medialen Welten und Netzen: Prozesse der Mediensozialisation.» In Handbuch Medien: Computerspiele; Theorie, Forschung, Praxis, hrsg. v. Jürgen Fritz u. Wolfgang Fehr 31-57. Bonn: Bundeszentrale für politische Bildung.

Baudry, Jean-Luis. 1994. "Das Dispositiv: Metapsychologische Betrachtungen des Realitätseindrucks.» Psyche 48: 1047-1074.

Biermann, Ralf. 2009. «Die Bedeutung des Habitus-Konzepts für die Erforschung soziokultureller Unterschiede im Bereich der Medienpädagogik.» MedienPädagogik 17 (14. Aug.): 1-18. www.medienpaed.com/17/\#biermann0908.

Bohnsack, Ralf. 2003. Rekonstruktive Sozialforschung: Einführung in qualitative Methoden. Opladen: Leske \& Budrich.

Bourdieu, Pierre. 1982. Die feinen Unterschiede: Kritik der gesellschaftlichen Urteilskraft. Frankfurt am Main: Suhrkamp. 
Fehr, Wolfgang, Jürgen Fritz. 1995. Warum Computerspiele faszinieren. Weinheim u. München: Juventa Verlag.

Fromme, Johannes, Norbert Meder und Nikolaus Vollmer. 2000. Computerspiele in der Kinderkultur. Opladen: Leske+Budrich

Glogauer, Werner. 1993. Die neuen Medien verändern die Kindheit. Weinheim: Deutscher Studien Verlag.

Hartmann, Frank. 2003. Mediologie: Ansätze zu einer Medientheorie der Kulturwissenschaften. Wien: Facultas.

Iske, Stefan und Christian Swertz. 2005. «Methodologische Fragen der Verwendung von Bild-, Ton- und Textdaten zur Navigationsanalyse.» MedienPädagogik 9 (8. Juni): 1-17. www.medienpaed.com/9/\#iske_swertz0506.

Iske, Stefan, Alex Klein, Nadia Kutscher und Hans-Uwe Otto. 2007. «Virtuelle Ungleichheit und informelle Bildung: Eine empirische Analyse der Internetnutzung Jugendlicher und ihre Bedeutung für Bildung und gesellschaftliche Teilhabe.» In Grenzenlose Cyberwelt?, hrsg. v. Kompetenzzentrum informelle Bildung, 65-92. Wiesbaden: VS-Verlag.

Innis, Harold A. .1997. Kreuzwege der Kommunikation. Wien: Springer.

Kommer, Sven. 2010. Kompetenter Medienumgang? Eine qualitative Untersuchung zum medialen Habitus und zur Medienkompetenz von SchülerInnen und Lehramtsstudierenden. Leverkusen-Opladen: Budrich UniPress.

Leu, Hans-Rudolf. 1993. Wie Kinder mit Computern umgehen: Studie zur Entzauberung einer neuen Technologie in der Familie. München: Deutsches Jugendinstitut.

McLuhan, Marshall. 1994. Die magischen Kanäle - Understanding Media. Dresden u. Basel: Verlag der Kunst.

Meder, Norbert. 1998. "Neue Technologien und Erziehung/Bildung.»In Deutsche Gegenwartspädagogik, Bd. 3, hrsg. v. Michele Borrelli u. Jörg Ruhloff, 26-40. Hohengehren: Schneider-Verlag.

Mikos, Lothar. 2007. «Distinktionsgewinne - Diskurse mit und über Medien.» In Medien - Macht - Gesellschaft, hrsg. v. Johannes Fromme u. Burkhard Schäffer, 45-60. Wiesbaden: VS-Verlag.

Neuß, Norbert. 2000. «Medienbezogene Kinderzeichnungen als Instrument der qualitativen Rezeptionsforschung.» In Qualitative Kinder- und Jugendmedienforschung: Theorie und Methoden, hrsg. v. Ingrid Paus-Haase u. Bernd Schorb, 131-154. München: kopaed.

Niesyto, Horst. 2009. «Digitale Medien, soziale Benachteiligung und soziale Distinktion.» MedienPädagogik 17 (24. Juni): 1-19. www.medienpaed.com/17/\#niesyto0906.

Paus-Hasebrink, Ingrid. 2009. «Zur Relevanz von sozialer Ungleichheit im Kontext der Mediensozialisationsforschung.» MedienPädagogik 17 (19. Mai): 1-21. www.medienpaed .com/17/\#paus-hasebrink0905.

Parzer, Michael. 2008. Musikgeschmack in der Popularkultur. Dissertation: Universität Wien. http://othes.univie.ac.at/2255/.

Reichenbach, Roland. 2001. Demokratisches Selbst und dilettantisches Subjekt: Demokratische Bildung und Erziehung in der Spätmoderne. Münster: Waxmann. Zugriff 11.12.2014. http://www.ife.uzh.ch/research/ae/mitarbeitende2/reichenbachroland/Rei chenbach_-_Demokratisches_Selbst_und_dilettantisches_Subjekt.pdf. .

Schäffer, Burkhard. 2003. Generationen - Medien - Bildung: Medienpraxiskulturen im Generationenvergleich. Opladen: Leske und Budrich.

Süss, Daniel. 1998. «Kinder im Sog virtueller Realitäten.» In Kind sein in der Schweiz: Eine Kulturgeschichte der frühen Jahre. Zürich, hrsg. v. Paul Hugger, 435-440. Zürich: Offizin. 
Swertz, Christian. 2003a. «Vorüberlegungen zu einer tranzendentalkritischen Medientheorie.» Spektrum Freizeit 2: 81-88.

Swertz, Christian. 2003b. «Was das Medium mit dem Wissen macht. McLuhan und die Wissensorganisation.» Information Wissenschaft \& Praxis 54 (2): 99-105.

Swertz, Christian. 2004. «Was das Medium mit der sozialen Arbeit macht: Reflexionen zum Einsatz der Computertechnologie in der pädagogischen Praxis.» Bildung und Lernen Online, hrsg. v. Werner Schindler, 65-75. München: kopaed.

Swertz, Christian. 2009. «Medium und Medientheorien.»In Umwelten: Sozialpädagogik/ Medienpädagogik/Interkulturelle und Vergleichende Erziehungswissenschaft/Umweltpädagogik. Handbuch der Erziehungswissenschaft Band III/2, hrsg. v. Norbert Meder, Christina Allemann-Ghionda u. Uwe Uhlendorff, 751-780. Paderborn: Ferdinand Schöningh: Paderborn.

Wagner, Ulrike und Helga Theunert. 2007. «Konvergenzbezogene Medienaneignung in Kindheit und Jugend.» MedienPädagogik 14 (11.Dez.): 1-20. www.medienpaed.com /14/\#wagner_theunert0712.

Wijnen, Christine. In Druck. «Medienpädagogik in Österreich.» In Kultur- und Medienbildung im internationalen Vergleich, hrsg. v. Stephan Buchloh.

This work is licenced under the Creative Commons Attribution-Share Alike 3.0 Austria License. To view a copy of this licence, visit http://creativecommons.org/licenses/by-sa/3.0/at/ or send a letter to Creative Commons, 171 Second Street, Suite 300, San Francisco, California 94105, USA. 